\title{
Efecto de un recubrimiento comestible comercial sobre las características fisicoquímicas de frutos de guayaba (Psidium guajava L.) bajo condiciones de almacenamiento
}

\author{
Carlos F. González-Chavarro ${ }^{1 *}$, Victor C. Pulido-Blanco ${ }^{2}$, Dario C. Pantoja-Espinosa ${ }^{3}$ y Fernando Portilla-Fuentes ${ }^{4}$ \\ (1) Corporación Colombiana de Investigación Agropecuaria - AGROSAVIA, Centro de Investigación Tibaitatá sede \\ Cimpa - km 2 vía antigua a Cite - Barbosa, Santander, Colombia. (correo-e: cfgonzalez@agrosavia.co). \\ (2) Corporación Colombiana de Investigación Agropecuaria - AGROSAVIA, Centro de Investigación Tibaitatá, sede \\ Tunja, Calle 9 \#9-35 Oficina 902, Tunja, Boyacá, Colombia. (correo-e: vpulido@agrosavia.co). \\ (3) Corporación Colombiana de Investigación Agropecuaria - AGROSAVIA, Centro de Investigación La Libertad - km 17 \\ vía Villavicencio a Puerto Lopez, Meta, Colombia. (correo-e: dcpantoja@agrosavia.co). \\ (4) Facultad de Ciencias Agropecuarias, Universidad Pedagógica y Tecnológica de Colombia - UPTC, Avenida Central \\ del Norte 39-115, Tunja, Boyacá, Colombia. (correo-e: fernando.portilla@uptc.edu.co).
}

* Autor a quien debe ser dirigida la correspondencia.

Recibido Oct. 21, 2020; Aceptado Dic. 21, 2020; Versión final Feb. 12, 2021, Publicado Jun. 2021

\begin{abstract}
Resumen
El objetivo de esta investigación fue evaluar la aplicación del recubrimiento comestible comercial Proallium Brill囚 (RC) en frutos de guayaba (Psidium guajava L.) ecotipo "regional roja". El RC no ha sido estudiado y puede ser una alternativa de conservación post cosecha. Se empleó un diseño experimental completamente al azar con distintas concentraciones de RC: T0 $\left(0 \mathrm{~g} \mathrm{l}^{-1}\right)$, T1 $\left(7 \mathrm{~g} \mathrm{l}^{-1}\right)$, T2 $\left(14 \mathrm{~g} \mathrm{l}^{-1}\right)$ y T3 $\left(21 \mathrm{~g} \mathrm{l}^{-1}\right)$. Las características fisicoquímicas fueron evaluadas a los 1,9 y 16 días después de almacenamiento (dda). El RC modificó los cambios fisicoquímicos, sin embargo, al 16 dda, el T3 presentó efectos estadísticamente significativos en la conservación de firmeza (65\%), mitigación de pérdida de peso (90.6\%), luminosidad y parámetro de color b. El T3 no influyó en índice de color, acidez total titulable, índice de madurez, ni tasa respiratoria. Se concluye que el tratamiento RC T3 logró conservar la firmeza de frutos de guayaba y minimizó la pérdida de peso.
\end{abstract}

Palabras clave: guayaba; recubrimiento comestible; Proallium Brill; post cosecha; almacenamiento de frutas.

\section{Effect of a commercial edible coating on the physicochemical characteristics of guava fruits (Psidium guajava L.) under storage conditions}

\begin{abstract}
The objective of this research study was to evaluate the application of the edible coating Proallium Brill囚 (EC) in guava (Psidium guajava L.) fruits (ecotype "regional roja"). The EC has never before been tested as a postharvest conservation alternative. Randomized sampling was used to test different EC concentrations: T0 $\left(0 \mathrm{~g} \mathrm{l}^{-1}\right)$, T1 $\left(7 \mathrm{~g} \mathrm{l}^{-1}\right)$, T2 $\left(14 \mathrm{~g} \mathrm{l}^{-1}\right)$, and T3 $\left(21 \mathrm{~g} \mathrm{l}^{-1}\right)$. The physicochemical characteristics were assessed at 1, 9 and 16 days after RC application and storage (das). The results showed that the RC treatment caused physicochemical changes. However, at 16 das, T3 showed significant statistical effects on firmness (65\%), mass loss mitigation (90.6\%), luminosity, and b color. T3 did not influence color index, titratable total acidity, maturity index, or respiratory rate. It is concluded that the RC T3 treatment preserves guava fruit firmness while minimizing weight loss.
\end{abstract}

Keywords: guava; edible coating; Proallium Brill; postharvest; fruit storage. 


\section{INTRODUCCIÓN}

La guayaba (Psidium guajava L.) es una fruta originaria de América Central y el Caribe, perteneciente a la familia Myrtaceae, distribuida en el trópico y subtrópico alrededor del mundo. Los mayores productores a nivel mundial en orden descendente son Brasil, India, Sur del África, México, Colombia, Cuba, Kenia y Taiwán (Hong et al., 2012). Por su parte en Colombia, los principales departamentos productores de guayaba son Meta (con una producción de 140845 t), Santander (32934 t), Boyacá (44200t), Valle del Cauca (18396 t) y Tolima (10044 t). En el departamento del Meta existen aproximadamente 2500 hectáreas sembradas, lo que representa una oportunidad productiva de desarrollo agroindustrial para la población del Piedemonte llanero y regiones como Lejanías, Granada, Acacías y Villavicencio (Aguilera-Arango et al., 2020).

Los frutos de guayaba se destacan entre los frutos tropicales no sólo debido a sus buenas características organolépticas (sabor y aroma) sino también nutricionales, son fuente de vitamina A, B1, B3 y C, fibra, minerales como potasio, calcio, hierro y fósforo. La guayaba roja tiene además un contenido relevante de licopeno, un importante carotenoide con propiedades antioxidantes que combaten la acción perjudicial de radicales libres en el organismo y permite contrarrestar enfermedades cardiovasculares (Achipiz et al., 2013). No obstante, la vida útil de la guayaba se limita de 2-3 días bajo condiciones ambientales del trópico, el proceso de maduración continúa de no emplearse un método poscosecha que minimice el riesgo de ablandamiento enzimático, degradación del color de la piel, generación de malos olores, proliferación de microrganismos y la degradación nutricional. Las pérdidas fluctúan entre el $5 \%$ y $25 \%$ en países desarrollados, mientras que en países en vía de desarrollo las pérdidas postcosecha ascienden al $80 \%$. Son evidentes las pérdidas económicas y el impacto social que ello implica, así como el impacto ambiental por la disposición de residuos orgánicos en depósitos dispuestos a la intemperie.

Considerando la alta perecibilidad de la guayaba usualmente se emplea contenedores plásticos para facilitar el almacenamiento y transporte, sin embargo, el uso de estos materiales con llevan impactos ambientales por la acumulación de residuos (Francisco et al., 2020). También se reporta la inmersión en soluciones de benomil, tiabendazol o imazalil para preservar frutos de guayaba, sin embargo, estos productos resultan peligrosos tanto para el consumo humano como para el medio ambiente (Biswas Murmu \& Niwas Mishra, 2018). Ante esta perspectiva, el uso de recubrimientos comestibles corresponde a una alternativa innovadora de conservación de frutas al disminuir parcialmente su tasa de respiración y procesos de maduración sin alterar la calidad organoléptica y composicional del material vegetal. Este método de conservación corresponde básicamente a la modificación de la microatmósfera del fruto, elevando la concentración de $\mathrm{CO}_{2}$ y disminuyendo el $\mathrm{O}_{2}$, lo que permite un intercambio gaseoso controlado, así como la disminución de la tasa metabólica y la humedad. Los recubrimientos comestibles poseen un gran rango de aplicabilidad y facilita la inclusión de ingredientes como compuestos antimicrobianos, antioxidantes, minerales, vitaminas, proteínas y demás compuestos de interés funcional (García-Mera et al., 2017).

Se han evaluado distintas formulaciones de recubrimientos comestibles en la evolución de la calidad poscosecha de guayaba, teniendo en cuenta características fisicoquímicas peso, ph, acidez, sólidos solubles totales, color, firmeza, entre otros parámetros a lo largo del almacenamiento. Los recubrimientos que se reportan en literatura son formulados a partir de quitosano, ácido cítrico (Idalice Gois Nascimento et al., 2020), aloe vera, extracto de ajo, jengibre, goma arábiga (Anjum et al., 2020), hidroxipropilmeltilcelulosa, cera de abejas, entre otros (Formiga et al., 2019) y se han obtenido resultados prometedores en la pérdida de peso, control en la proliferación de hongos, reducción de pardeamiento y alteraciones de color, conservación de firmeza, actividad antioxidante y en general preservación de la calidad y prolongación de la vida útil de frutos de guayaba. No obstante, no se reportan estudios empleando el producto comercial Proallium Brill, un producto disponible en el mercado cuya composición incluye lecitina, ácido ascórbico, ácido cítrico y demás ingredientes. Teniendo en cuenta lo anterior, el objetivo del estudio fue estudiar el efecto de la aplicación del producto comercial Proallium Brill $\circledast$ en características fisicoquímicas de frutos guayaba ecotipo regional roja (Psidium guajava L.), genotipo cultivado ampliamente en Colombia que necesita agroindustrialización para garantizar la sostenibilidad económica del cultivo y la articulación con pequeños industriales del país y la región.

\section{MATERIALES Y METODOS}

La materia prima empleada en el estudio, así como el diseño de experimentos, metodología de pruebas fisicoquímicas y análisis estadístico, se describen en las siguientes partes: material biológico, preparación y aplicación de cera comestible, diseño experimental, determinación de firmeza, pérdida de masa, índice de color del fruto, acidez total titulable, sólidos solubles totales, índice de madurez, tasa respiratoria, y análisis estadístico.

Material biológico: Se utilizaron frutos de guayaba ecotipo Regional Roja obtenidos de los árboles elite del Centro de Investigación Tibaitata sede Cimpa (Centro de Investigación para el Mejoramiento de la 
Agroindustria Panelera), ubicado $5^{\circ} 56^{\prime} 5^{\prime \prime} \mathrm{N}$ y $73^{\circ} 36^{\prime} 24^{\prime \prime} \mathrm{O}$ en la subregión Hoya del Rio Suárez (HRS), municipio de Barbosa (Santander) a $1650 \mathrm{msnm}$, temperatura promedio de $18-24{ }^{\circ} \mathrm{C}$ y precipitación media anual de $1.600 \mathrm{~mm}$. La recolección se realizó de forma manual, en estado de maduración pintón, escala de color dos (2) verde claro tendiendo a amarillo verdoso brillante (Solarte et al., 2010), los frutos se tomaron de la parte media del árbol, fueron colocados en una cesta con superficie lisa, posteriormente fueron empacados en bolsas plásticas para ser transportadas al laboratorio de fisiología vegetal de la Facultad de Ciencias Agropecuarias de la Universidad Pedagógica y Tecnológica de Colombia “UPTC" (Tunja, Boyacá), y luego los frutos fueron desinfectadas con una solución de hipoclorito de sodio $(\mathrm{NaCl})$ al $5 \%$, en una bandeja plástica de $6 \mathrm{~L}$.

Diseño experimental: El diseño experimental utilizado fue completamente al azar (DCA) teniendo en cuenta lo definido en estudios previos (García-Mera et al., 2017). Se emplearon cuatro (4) tratamientos como dosis de aplicación de RC Proallium Brill $囚$, tratamiento T0 (Testigo), sin cera, tratamiento uno (T1) con una concentración de $7 \mathrm{~g} \mathrm{l}^{-1}$, tratamiento dos (T2) concentración $14 \mathrm{~g} \mathrm{l}^{-1} \mathrm{y}$ tratamiento tres (T3) concentración 21 $\mathrm{gl}^{-1}$, cada tratamiento estuvo compuesto por 4 frutos como unidad experimental, se llevó a cabo por triplicado para un total de 48 frutos.

Preparación y aplicación de cera comestible: Se emplearon 4 frutos de materia prima y se realizó la aplicación del recubrimiento comestible $(\mathrm{RC})$ marca Proallium Brill $\circledast$ según la formulación de cada tratamiento T0, T1, T2 y T3. EI RC se preparó conforme a indicaciones del fabricante y su aplicación se realizó mediante inmersión de frutos por un tiempo de 10 minutos en una bandeja plástica de 3 litros de capacidad. Una vez se dispuso del material tratado, se almacenó a condiciones ambientales en un lugar aséptico previamente dispuesto en el laboratorio de fisiología vegetal de la UPTC, temperatura promedio de $12.9^{\circ} \mathrm{C}$ y humedad relativa de $85.5 \%$. La concentración del RC correspondió a la variable independiente, mientras que las propiedades fisicoquímicas correspondieron a las variables dependientes: firmeza, peso fresco, pérdida de masa, índice de color, acidez titulable, sólidos solubles totales, índice de madurez y tasa de respiración. Los parámetros se definieron conforme a metodologías previas (Balaguera-López et al., 2019)

Firmeza: Se determinó con un penetrómetro digital PCE-PTR200 (PEC Ibérica S.L., Albacete, España), provisto de un pistón de $8 \mathrm{~mm}$, la medición en cada fruto se hizo sobre la parte ecuatorial, de forma perpendicular, ejerciendo una presión que deforme los frutos, $60 \mathrm{~N}$ (Azzolini et al., 2005), siendo la misma persona quien realizó todas las mediciones; los resultados se expresaron en Newtons $(1 \mathrm{~kg}=9.8 \mathrm{~N})$.

Pérdida de masa: Se determinaron por gravimetría empleando una balanza (Ohaus Ohio, $\mathrm{OH}$ ) de precisión $0,00001 \mathrm{~g}$. Se registraron los pesos de cada fruta en el día 0 (peso inicial), menos la diferencia en las diferentes fechas de muestreo, hasta el día final de evaluación. Las pérdidas acumuladas de peso se expresaron como porcentaje de pérdida de peso (\%), mediante la ecuación (1):

$\%$ Pérdida de peso $=(($ Peso inicial - Peso final $)) /($ Peso inicial $) \times 100$

Índice de color del fruto (IC): Se utilizó un colorímetro triestímulo Minolta (Osaka, Japón), cada fruto tuvo tres lecturas en el diámetro ecuatorial. Los resultados obtenidos se expresan como coordenadas de color CIELAB, es decir, como parámetros $L^{*}, a^{*}$ y $b^{*}$ (valores de Hunter) donde, $L^{*}$, es el eje perpendicular al plano y va de oscuro a claro, llamado luminosidad ( $0-100)$, $a^{*}$, es el eje de las abscisas y va de verde a rojo $(-a,+a) ;(-60,+60)$ y $b^{*}$, es el eje de ordenadas y va de azul a amarillo $(-b,+b) ;(-60,+60)$. El índice de color (IC) se calculó según la ecuación (2):

$\mathrm{IC}=\mathrm{a} / \mathrm{L} \times \mathrm{b} \times 1000$

Acidez total titulable (ATT): Se determinó mediante la valoración con $\mathrm{NaOH} 0.1 \mathrm{~N}$ a partir $1 \mathrm{ml}$ del zumo de una fruta diluido en $50 \mathrm{ml}$ de agua destilada, se tituló con $\mathrm{NaOH}$ y el indicador de fenolftaleína al $0.1 \%$, los resultados se expresaron en porcentaje de ácido cítrico en guayaba y se calculó mediante la ecuación (3):

$\%$ Ácido cítrico $=$ Vol. $\mathrm{NaOH}$ gastado $(\mathrm{ml}) \times$ Normalidad $\mathrm{NaOH}(0.1) \times$ Meq. ácido cítrico $(0.067) \times$

100 mililitros de la muestra

Sólidos solubles totales (SST): Se obtuvo jugo de guayaba en agua (relación $1 / 4$ ) debidamente filtrado para analizar los SST. Para ello se empleó un refractómetro digital Hanna (Hanna Instruments, Woonsocket, RI) de rango 0 a $85 \%$ que realiza medición compensando la temperatura a $20^{\circ} \mathrm{C}$. Los resultados se expresaron en ${ }^{\circ}$ brix (Achipiz et al., 2013).

Índice de madurez (IM): Se obtuvo mediante la relación entre solidos solubles totales ( ${ }^{\circ}$ brix) con la acidez total titulable (ATT), se calculó con la ecuación (4):

$\mathrm{IM}=\mathrm{SST} / \mathrm{ATT}$ 
Tasa respiratoria (TR): Se realizó mediante un sensor infrarrojo de $\mathrm{CO}_{2}$ con cámaras herméticas de $2 \mathrm{~L}$, el cual se conectó a un sistema de interfaz y recolección de datos marca Labquest 1 , este equipo determina la cantidad de mg CO $\mathrm{kg}^{-1} \mathrm{~h}^{-1}$. Para hacer la medición al fruto, fue dispuesto en la cámara junto con el sensor infrarrojo, se tomaron intervalos de 4 segundos entre puntos y durante 5 minutos en los cuales se registraron los valores de $\mathrm{CO}_{2}$, con estos valores se calculó la pendiente, que correspondió a la tasa respiratoria. Se tuvo en cuenta el peso del fruto $(\mathrm{kg})$ y el volumen de la cámara para convertir los datos a mg CO $2 \mathrm{~kg}^{-1} \mathrm{~h}^{-1}$.

Análisis estadístico: Los datos obtenidos fueron sometidos a prueba de normalidad, mediante Shapiro-Wilk y de homogeneidad de varianzas, mediante Levene. Comprobados los supuestos, se realizó el análisis de varianza (ANAVA) con un intervalo de confianza de $95 \%(\alpha=5 \%)$, mediante el software sistema de análisis estadístico (SAS) versión 9.4.

\section{RESULTADOS Y DISCUSIÓN}

La firmeza en los frutos de guayaba, a los 1 y 9 días después de almacenamiento (dda), no presentaron diferencias estadísticamente significativas entre tratamientos $(P \geq 0,05)$, su valor fluctuó entre $46.9 \mathrm{~N}$, T3 y 57.2 N, T0 (Figura 1). Posteriormente a los 9 dda, se presentó una reducción a un 17.9\% para el T1, 19\% para T0 y $30.7 \%$ para T3, por lo cual se puede inferir que a medida que el fruto madura, la firmeza disminuye. Los resultados concuerdan con lo indicado por varios autores (Azzolini et al., 2005; Solarte et al., 2010).

A los 16 dda, hubo diferencias estadísticamente significativas $(P \leq 0,05)$, siendo el mejor tratamiento el T3 con $21.4 \mathrm{~N}$, presentando mayor resistencia, seguido por T2 con $13.4 \mathrm{~N} \mathrm{y}$, por último, $7.8 \mathrm{~N}$ en T0 (Figura 1). El comportamiento presentado se relaciona con la formación de una barrera superficial en el fruto generada por el recubrimiento comestible, que modifica la composición gaseosa interna. Este fenómeno disminuye la tasa de respiración y la degradación de compuestos de la pared celular, causada por el aumento en la actividad de enzimas hidrolasas, que actúan sobre la pectina de la pared celular, las pectinasas, remueven los grupos metilo del ácido galacturónico, permitiendo a las poligalacturonasas, despolimerizar la cadena de ácido poligalacturónico, reduciendo así su peso molecular, también están implicadas las celulasas, las cuales incrementan conforme madura el fruto (Solarte et al., 2010).

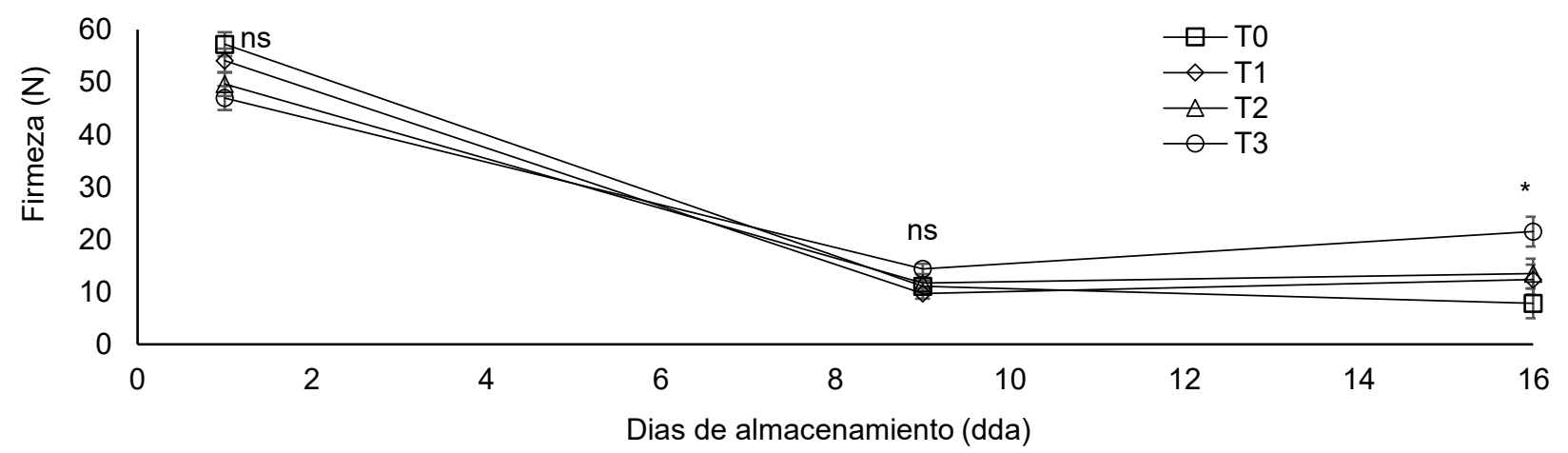

Fig. 1: Firmeza a diferentes concentraciones de cera comestible Proallium Brill@. Diferencias significativas al $5 \%\left({ }^{*}\right)$ y no hay diferencias (ns), de acuerdo con ANAVA. Barras verticales indican error estándar $(n=4)$.

La pérdida de peso del fruto aumentó a medida que avanzó el tiempo poscosecha, sin embargo, a los 16 dda el T3, evidencia diferencias estadísticamente significativas $(P \leq 0,05)$ con respecto a los otros tratamientos, el T3 presentó un $9.4 \%$ de pérdida mientras que el T0 tuvo la mayor pérdida, con $27.6 \%$ (Figura 2), lo que indica la influencia de mayor concentración de recubrimiento sobre la pérdida de peso, los frutos de guayaba recubiertos perdieron menos peso que los frutos que estaban sin recubrimiento, resultados similares han sido reportados en frutos de guayaba, donde sus pérdidas oscilaron entre $4.2 \%$ y $11.2 \%$, para los ecotipos de guayaba Guavatá, Ráquira, Regional Roja y Blanca (Solarte et al., 2010), Castellano et al. (2005) evaluando $\mathrm{Cl}_{2} \mathrm{Ca}$, obtuvo las menores pérdidas de peso al utilizarlo en una concentración del $2 \%$, Achipiz et al. (2013) investigando en una cera a base almidón consiguió el mejor comportamiento al 4\% y González et al. (2016), obtuvo pérdidas entre $0,92 \%$ y $4,31 \%$, utilizando recubrimiento al $4 \%(\mathrm{p} / \mathrm{v})$ de proteína de suero de leche concentrado y $10 \%(\mathrm{p} / \mathrm{v})$ de glicerina. Esta pérdida de peso se atribuye al aumento en la transpiración y respiración, pérdida de agua y compuestos volátiles, además de ser la principal causa de deterioro, debido a que origina pérdidas directas sobre la calidad del fruto, pérdida de brillo, turgencia y valor nutricional; y que por ende tiene valores aceptables entre 10\% y 15\% (Becerra et al., 2009). Amarante et al. (2001), afirman que el recubrimiento o cera reduce la permeabilidad al vapor de agua y el intercambio gaseoso entre el fruto y su entorno, ya que la cubierta externa bloquea los poros de la epidermis y se logra una reducción en la pérdida de agua de los tejidos retrasando la aparición de síntomas por marchitamiento. 


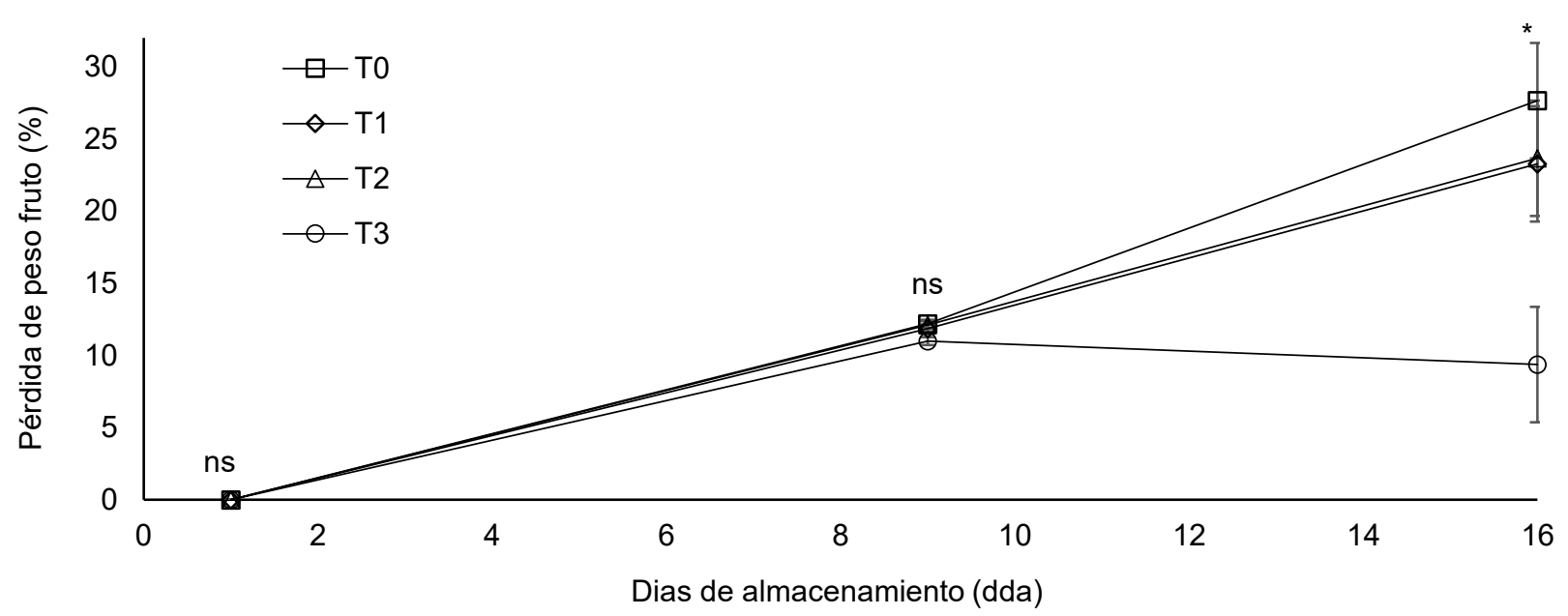

Fig. 2: Pérdida de peso del fruto a diferentes concentraciones de cera Proallium Brill@. Diferencias significativas al $5 \%\left({ }^{*}\right)$ y no hay diferencias ( $n s)$, de acuerdo con ANAVA. Barras verticales indican error estándar $(n=4)$.

EI IC fluctuó entre los valores -5 y 10 , lo cual se ubica entre índices de colores verde amarillento, amarillo verdoso y amarillo con tonalidades naranja leves (Vignoni et al., 2006). El parámetro describió una tendencia creciente durante el período de almacenamiento, presentando diferencias estadísticamente significativas entre tratamientos $(P \leq 0,05)$ a los 9 dda; sin embargo, no se presentaron diferencias al final de la evaluación. La luminosidad presentó diferencias estadísticamente significativas $(P \leq 0,05)$ a los 9 dda, tendiendo a un color levemente oscuro con valores aproximados de 68 para el T3 y T0 mientras que el T1 y T2 obtuvieron 72 y 76 respectivamente. El parámetro se considera claro, debido a que $L^{*}$, tiende a 0 cuando el color es oscuro y a 100 cuando es claro. Finalmente, a los $16 \mathrm{dda}$, se obtuvo diferencias estadísticamente significativas $(P \leq 0,05)$, entre el T3, con 43 y los otros tratamientos, el T3 adquirió tonalidades oscuras mientras que los tratamientos T0, T1 y T2, tuvieron valores entre 63 y 71, siendo mayormente claros. Cabe aclarar que dentro de los objetivos no se consideró la medición de los parámetros Hue y croma, puesto que se buscó registrar el cambio de color de la fruta, no definir la pureza o saturación de este. El índice de color es un parámetro válido para llevar a cabo un registro de la variación del color de frutos durante su almacenamiento.

En relación con el parámetro $\left(\mathrm{a}^{*}\right)$, cabe resaltar que para el estado 2 de maduración, la coloración del fruto fue del $50 \%$ verde y $50 \%$ amarillo, lo cual se evidencia al 1 dda para todos los tratamientos. El parámetro $\left(a^{*}\right)$ presentó únicamente diferencias estadísticamente significativas $(P \leq 0,05)$ a los 9 dda, el T1 tuvo el menor valor con 4 , interpretándose como levemente rojizo y T0 (con 14, es decir con una coloración rojiza más intensa. A medida que el valor del parámetro $\left(a^{*}\right)$ es positivo, el color tiende a rojo y, por el contrario, si es negativo tiende a verde, por consiguiente, es lógico que el T0, presente tonalidad rojiza debido a la ausencia de recubrimiento.

Por su parte, los valores del parámetro $\left(b^{*}\right)$, coinciden también con el estado 3 de maduración, a los 16 dda, el T3, presentó diferencias estadísticamente significativas $(P \leq 0,05)$, tomando valores de 25 , interpretados como coloración amarilla oscura. Por otra parte, los otros tratamientos describieron valores que fluctuaron entre 36 y 42, interpretándose como coloraciones amarillas claras. Si el parámetro $\left(b^{*}\right)$ toma valores positivos, corresponde al color amarillo y, por el contrario, si toma valores negativos, corresponde al color azul (Figura 3). Los resultados permiten inferir que, a mayor contenido de recubrimiento, menor es el valor de $b^{*}$, así que referido a valores positivos se puede estimar que la coloración amarillo oscuro, comprende valores de 0 a +30 y claro de $+30 \mathrm{a}+60$.

González (2010), reportó cambios de color externo a* para guayaba variedad Palmira en tres estados de madurez, $-0.21 \pm 1.45$ para frutos $100 \%$ verdes, $5.90 \pm 2.31$ en frutos $50 \%$ amarillos y $8.88 \pm 1.09$ en $100 \%$ amarillos, recalcando que la coordenada $a^{*}$ es el parámetro que más se afecta a la maduración ya que va de valores negativos, tonos verdosos de la fruta, a valores positivos para tonos rojizos, este cambio de color es favorecido por la degradación de la clorofila y por la síntesis de otros pigmentos como antocianinas y carotenoides, este mismo autor concluye que en guayaba el cambio de color indica madurez en poscosecha.

Los valores de ATT aumentaron durante la evaluación, cambiando de $0.55 \%$ a $1.09 \%$ para T0 y $0.85 \%$ a 1.01 para T2, desde el 1 dda hasta los 16 dda (Figura 4). Se presentaron diferencias estadísticamente significativas $(\mathrm{P} \leq 0,05)$ al $1 \mathrm{dda}$, teniendo T2 la mayor acidez y T0 la menor acidez presentada; a los 9 dda, también se detectaron diferencias estadísticamente significativas $(P \leq 0,05)$, teniendo el $\mathrm{T0} 1.04 \%$ y el T2, $1 \%$, conformando un solo grupo con la mayor acidez, la menor acidez se halló en T1 con $0.84 \%$ y un valor intermedio con $0.89 \%$ para el T3 (Figura 4 ). 
(a)

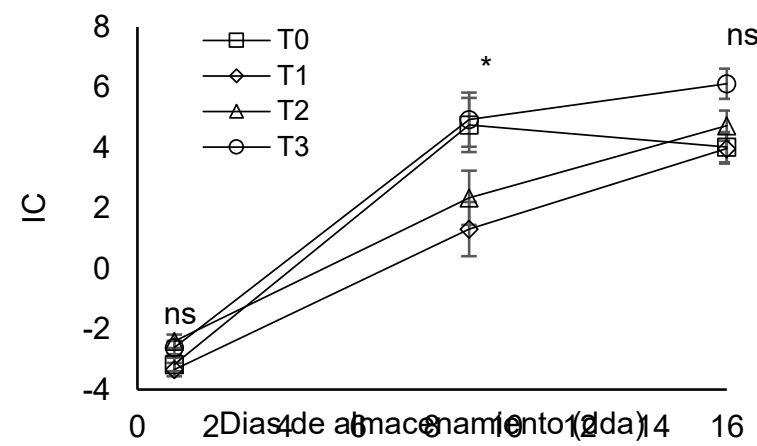

(c)

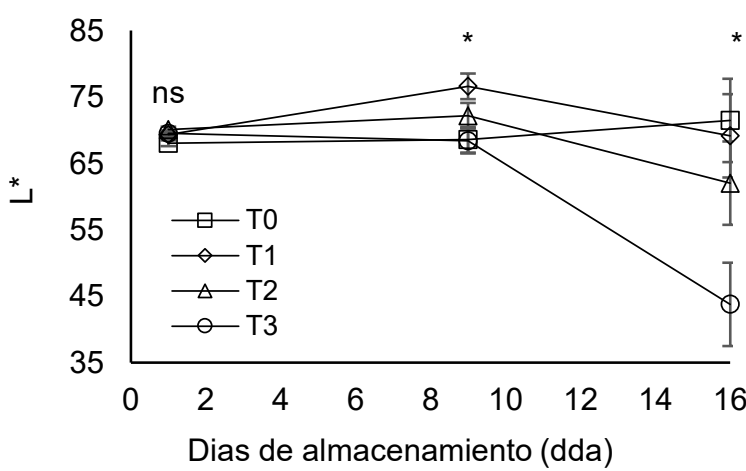

(b)

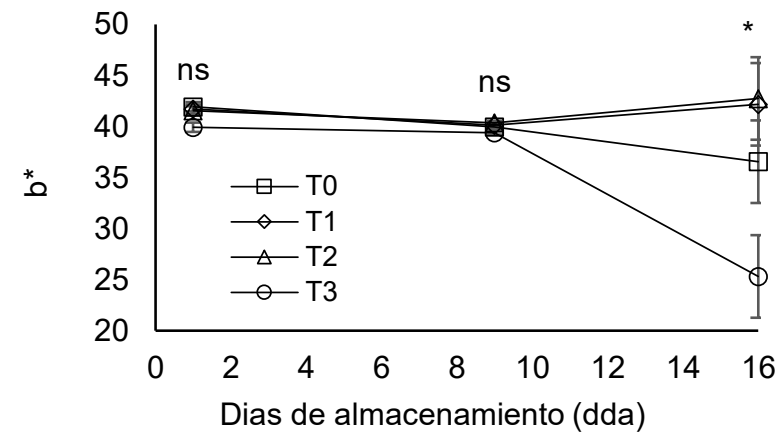

(d)

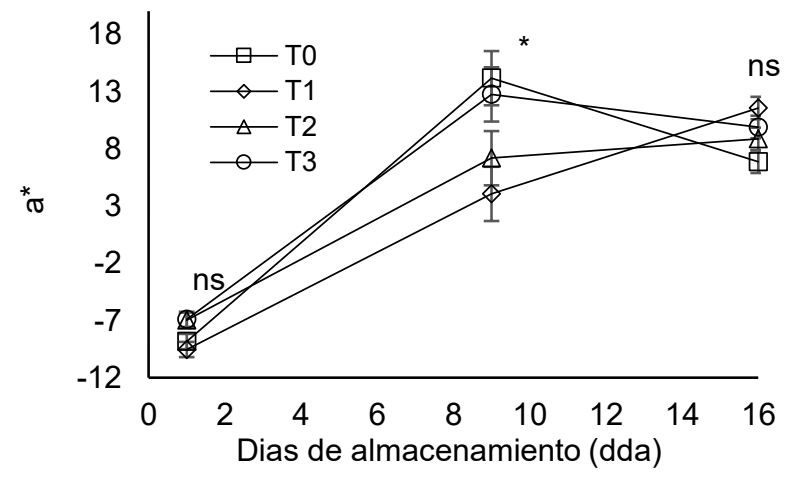

Fig. 3: A, B Índice de color (IC); C, D. luminosidad $\left(L^{*}\right)$, abscisas $\left(a^{*}\right)$ y ordenadas $\left(b^{\star}\right)$ durante almacenamiento. Diferencias significativas al $5 \%\left(^{*}\right)$ y no hay diferencias (ns), de acuerdo con ANAVA. Barras verticales indican error estándar (n=4).

Estos resultados demuestran que el comportamiento de la ATT durante la maduración no es coincidente con la literatura citada puesto que se reporta el aumento de ATT con el máximo en el climatérico, seguido de una subsecuente disminución (Bashir y Abu-Goukh, 2003), reducciones o estabilización del parámetro (Cavalini et al., 2006; Solarte et al., 2010). La reducción se atribuye a la utilización de ácidos orgánicos como sustrato en la respiración, por el contrario, el aumento permite suponer una baja tasa de respiración que limita el consumo de reservas en los frutos recubiertos (Restrepo-Sánchez et al., 2009).

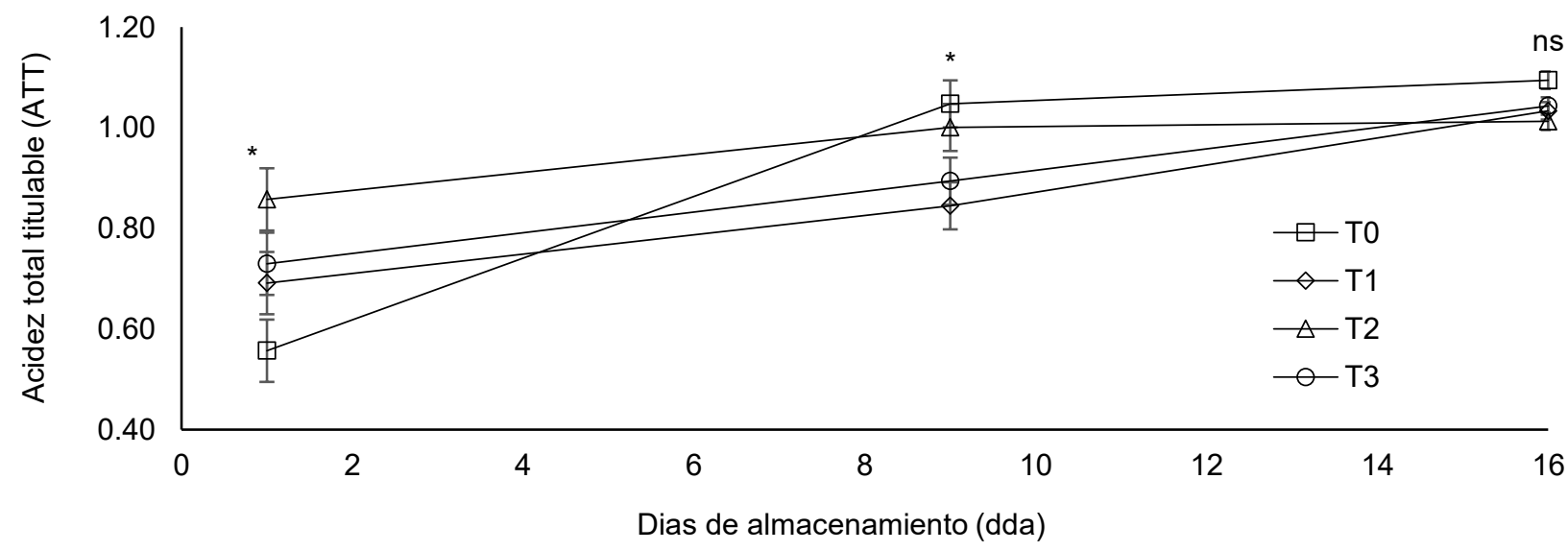

Fig. 4: Acidez total titulable a diferentes concentraciones de cera comestible Proallium Brill $5 \%\left(^{*}\right)$ y no hay diferencias (ns), de acuerdo con ANAVA. Barras verticales indican error estándar $(n=4)$.

Los SST en frutos de guayaba recubierta tuvieron una disminución constante durante la evaluación, al 1 dda, se presentaron diferencias estadísticamente significativas $(P \leq 0,05)$, el mayor valor lo presentó el T3 con 13.3 'brix y el menor T1 con $9.8^{\circ}$ brix (Figura 5), a los 16 dda, se presentaron diferencias estadísticamente significativas $(P \leq 0,05)$, teniendo la mayor disminución el T2 con $49.3 \%$ y la menor el T1 con $18.1 \%$ (Figura 
5); este comportamiento puede ser debido a la disminución de carbohidratos, los cuales forman parte de la estructura de las células y funcionan como reserva de energía (Restrepo-Sánchez et al., 2009; Solarte et al., 2010).

Figueroa et al. (2011), afirman que la disminución de los SST en los frutos recubiertos puede estar relacionado con la menor tasa de respiración exhibida, que afectan la síntesis y degradación de compuestos de reserva en el ciclo de Krebs, que en el caso de los frutos son los azucares y los ácidos orgánicos. Los resultados coinciden con lo obtenido en fresa y guayaba, fenómeno atribuido al proceso de respiración y actividad metabólica, lo cual redujo la formación y uso de metabolitos propios del proceso de maduración como la hidrólisis de almidón (Gol et al., 2013; Idalice Gois Nascimento et al., 2020; Rehman et al., 2020). Igualmente existe coincidencia con lo reportado por Castellano et al. (2005), quienes obtuvieron una reducción de SST en frutos recubiertos con $\mathrm{Cl}_{2} \mathrm{Ca}$ sumergidos en agua a temperatura ambiente y caliente a $45^{\circ} \mathrm{C}$ por 5 minutos. Ahora bien, el fenómeno presentado en la muestra control sugiere que las frutas presentaron procesos de fermentación (de Oliveira et al., 2020).

Por otra parte, los resultados obtenidos difieren con lo indicado por varios autores que hallaron un aumento en los SST durante la poscosecha, con valores que oscilaron de 5.0 a $13.2^{\circ}$ brix (Bashir y Abu-Goukh, 2003), Solarte et al. (2010) obtuvieron variaciones entre 8.6 y $12^{\circ}$ brix en los ecotipos Regional Roja, Blanca, Ráquira y Guavatá victoria, con aumento en el IM, lo que les permitió inferir un necesario aumento de carbohidratos acompañado de una reducción de ácidos orgánicos mientras transcurre la maduración en poscosecha, y finalmente González (2010) reporta un comportamiento similar para los ecotipos Guayaba Roja, Blanca y Palmira ICA-1.

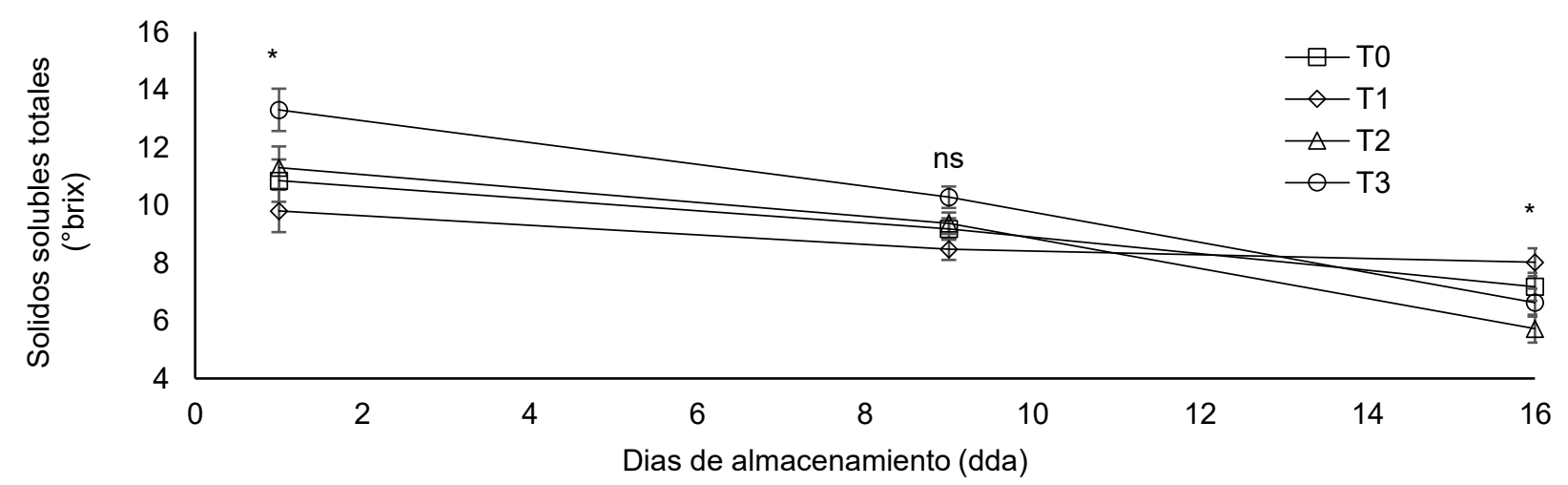

Fig. 5: Solidos solubles totales a diferentes concentraciones de cera comestible Proallium Brill. Diferencias significativas al $5 \%\left({ }^{*}\right)$ y no hay diferencias (ns), de acuerdo con ANAVA. Barras verticales indican error estándar $(n=4)$.

Durante la evaluación, el IM presentó diferencias estadísticamente significativas $(P \leq 0,05)$ al 1 dda, siendo el mayor valor el obtenido por T0 con 18.5 y T3 con 18.2, sin embargo, aunque no se presentaron diferencias estadísticamente significativas $(P \geq 0,05)$ a los 9 dda y $16 \mathrm{dda}$, los tratamientos presentaron una disminución promedio del $39.6 \%$ y $58.4 \%$, respectivamente (Figura 6). Esta reducción se debió a la relación directa que existe entre el IM y los SST que describieron una disminución, y la relación indirecta con la ATT, que aumentó durante el almacenamiento. La tendencia del IM no se reporta en estudios previos, pero evidencia la reducción de la velocidad de reacciones de maduración (Arroyo et al., 2020). En investigaciones realizadas por Gutiérrez Guzmán et al. (2012), el parámetro IM toma valores comprendidos entre 8 a 18, incluso valores superiores a 30 dependiendo del material vegetal; no obstante, el consumo de guayaba no requiere que la fruta alcance valores altos de IM. Los valores presentados coinciden con los reportados para guayabas de la variedad "Pedro Sato" (Azzolini et al., 2005); para otras variedades de guayaba, el rango esta entre 8 y 18 (Andrade et al., 2009; Silva et al., 2006), en los ecotipos de la HRS, se obtuvo lo siguiente, Guayaba Regional, Blanca 18.83 y Roja 17.3, Ráquira blanca 21.38 y Guavatá Victoria 12.57 (Solarte et al., 2010).

La TR no presentó diferencias estadísticamente significativas $(P \geq 0,05)$, entre los tratamientos; sin embargo, al $1 \mathrm{dda}$, tuvo un promedio de $59.59 \mathrm{mg} \mathrm{CO}_{2} \mathrm{~kg}^{-1} \mathrm{~h}^{-1}$, posteriormente se redujo en promedio a $42.53 \mathrm{mg} \mathrm{CO}_{2}$ $\mathrm{kg}^{-1} \mathrm{~h}^{-1}$ y a los $16 \mathrm{dda}$, para el T0 y T3, disminuyó en promedio a $24.6 \mathrm{mg} \mathrm{CO}_{2} \mathrm{~kg}^{-1} \mathrm{~h}^{-1}$, mientras que para T1 y T2, su disminución fue muy leve con un valor de $37.7 \mathrm{mg} \mathrm{CO}_{2} \mathrm{~kg}^{-1} \mathrm{~h}^{-1}$, por tanto, se puede afirmar que hubo una disminución cuantiosa conforme transcurrieron los días de evaluación (Figura 7), este comportamiento se relaciona con la baja presencia de oxígeno y síntesis de metabolitos en el fruto, el cual es favorecido debido al alto poder de recubrimiento que ejerce el recubrimiento aplicado, la cual crea una barrera física sobre el fruto, reduciendo la tasa de respiración. 


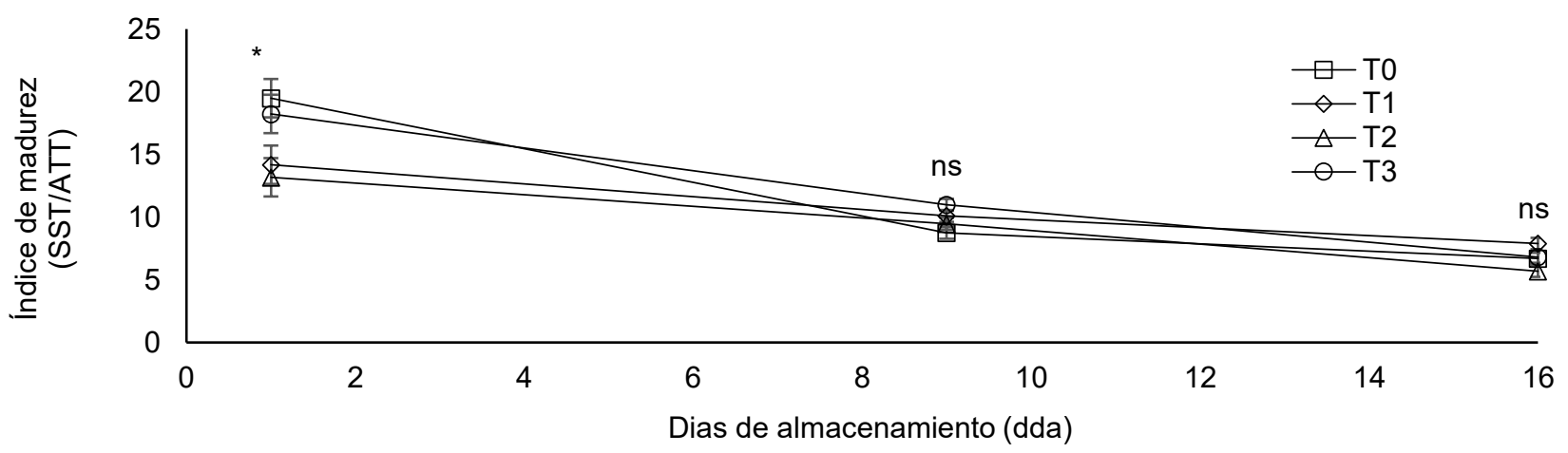

Fig. 6: Índice de madurez a diferentes concentraciones de cera comestible Proallium Brill. Diferencias significativas al $5 \%$ $\left({ }^{*}\right)$ y no hay diferencias (ns), de acuerdo con ANAVA. Barras verticales indican error estándar $(n=4)$.

Las investigaciones citadas indican que la acción de los RC se basa en la formación de una capa semipermeable que promueve la acumulación de $\mathrm{CO}_{2}$ y la reducción del $\mathrm{O}_{2}$, lo que conlleva a la supresión del etileno necesario para continuar con la maduración. Estos resultados no coinciden con los reportados en un estudio bajo condiciones de laboratorio, la crisis climatérica inicia a las 50 horas y el pico climatérico se presenta a las 60 horas, teniendo una producción entre 60 y $100 \mathrm{mg} \mathrm{CO}_{2} \mathrm{~kg}^{-1} \mathrm{~h}^{-1}$, limitando la vida útil del producto. La guayaba presenta un pico climaterio entre los 3 y $12 \mathrm{dda}$, y el comportamiento es fuertemente influenciado por la temperatura, las frutas de guayaba alcanzaron un pico climatérico a los 9 días con 138.27 $\mathrm{mg} \mathrm{CO} 2 \mathrm{~kg}^{-1} \mathrm{~h}^{-1}$ y $241 \mathrm{mg} \mathrm{CO}_{2} \mathrm{~kg}^{-1} \mathrm{~h}^{-1}$, para frutos almacenados a $10^{\circ} \mathrm{C}$ y $20^{\circ} \mathrm{C}$, respectivamente.

Varios autores consideran la clasificación de la guayaba como contradictoria, algunos autores la consideran fruto climatérico (Azzolini et al., 2005) o no climatérico (Singh y Pal, 2008; Solarte et al., 2010); esa característica puede ser un atributo varietal (Azzolini et al., 2005) y/o generado por condiciones ambientales (Solarte et al., 2010), las variedades climatéricas presentan tasa respiratoria entre 31 a $1.400 \mathrm{mg} \mathrm{CO}_{2} \mathrm{~kg}^{-1} \mathrm{~h}$ ${ }^{1}$, mientras que otros autores señalan que la fruta produce 70 a $150 \mathrm{mg} \mathrm{CO}_{2} \mathrm{~kg}^{-1} \mathrm{~h}^{-1}$, siendo un fruto climatérico moderado.

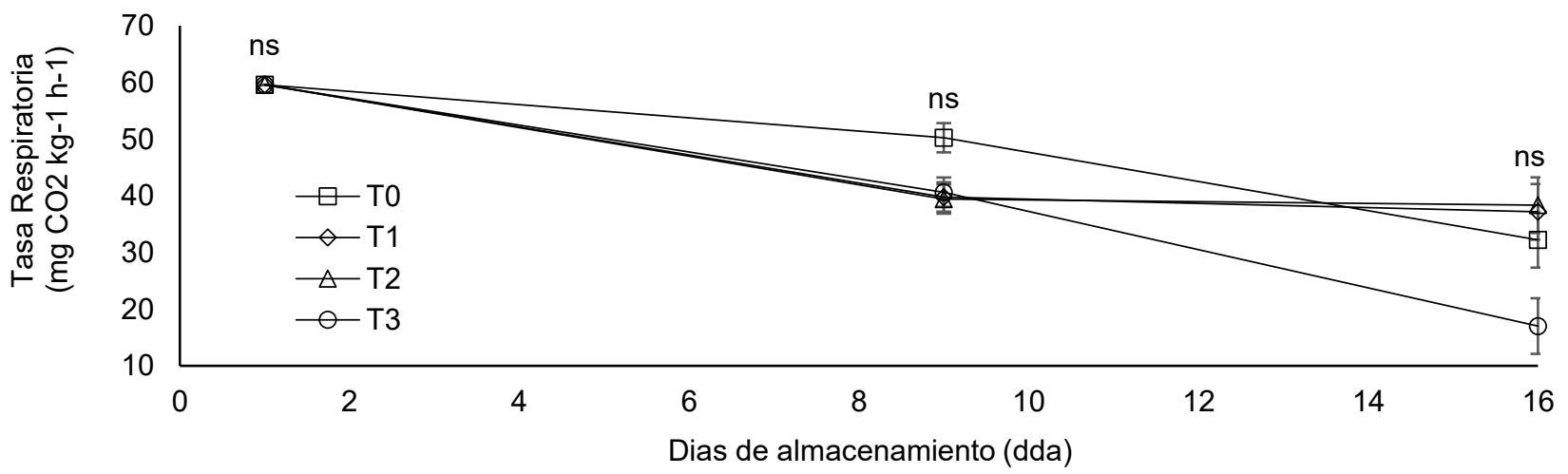

Fig. 7: Tasa respiratoria a diferentes concentraciones de cera comestible Proallium Brill. Diferencias significativas al $5 \%$ $\left({ }^{*}\right)$ y no hay diferencias (ns), de acuerdo con el ANAVA. Barras verticales indican el error estándar $(n=4)$.

\section{CONCLUSIONES}

El tratamiento T3 correspondiente a frutos los recubiertos con el RC Proallium Brill $囚$ a una concentración de $21 \mathrm{~g} \mathrm{l}^{-1}$, logró conservar la firmeza de frutos de guayaba y minimizó la pérdida de peso con diferencias estadísticamente significativas a los 16 dda. EI IC fue significativamente más alto en las muestras T3 a los 9 dda, pero no se evidenciaron diferencias a los 16 dda, día en el cual se registró una luminosidad y un parámetro $b$ significativamente menores con respecto al control y los otros tratamientos. De la misma forma a los $9 \mathrm{dda}$, se presentaron diferencias estadísticamente significativas en ATT de la muestra control, la cual fue mayor que la registrada en las muestras recubiertas. Los SST, IM y TR presentaron comportamientos inusuales con respecto a lo reportado en otras investigaciones. Los SST de la muestra control y las muestras recubiertas presentaron una tendencia decreciente a lo largo del almacenamiento, con diferencias estadísticamente significativas al final del período de estudio. Este fenómeno es atribuido a procesos metabólicos propios del fruto y a procesos fermentativos. Existe una relación directa de los SST con el IM, este último describió una disminución a lo largo del almacenamiento. La TR se redujo a lo largo del almacenamiento, sin presentar diferencias estadísticamente significativas entre tratamientos. 


\section{AGRADECIMIENTOS}

Los autores agradecen a las siguientes personas que participaron en el trabajo de campo y en el desarrollo del proyecto: Eugenio Kopp Sanabria y Javier Jimenez Vargas.

\section{REFERENCIAS}

Achipiz, S., Castillo, A. y otros tres autores, Efecto de recubrimiento a base de almidón sobre la maduración de la guayaba (Psidium guajava), Rev. Bio. Agro, 11(2), 92-100 (2013)

Aguilera-Arango, G., Rodríguez-Henao, E. y otros tres autores, Estado actual de la investigación para el cultivo de guayaba en Colombia, https://doi.org/10.15517/am.v31i3.40207, Agron. Mesoam, 31(3), 845-860 (2020)

Amarante, C., Banks, N. y Ganesh, S., Effects of coating concentration, ripening stage, water status and fruit temperature on pear susceptibility to friction discolouration, https://doi/10.1016/S0925-5214(00)00155-1, Postharvest Biol. Technol, 21(3), 283-290 (2001)

Andrade, P., Ortega, Q. y otros cinco autores, Caracterización fisicoquímica y reológica de la pulpa de guayaba (Psidium guajava L.) variedades híbrido de Klom Sali, Puerto Rico, D14 y Red. Vitae, 16(1), 13-18 (2009)

Anjum, M., Akram, H., Zaidi, M. y Ali, S., Effect of gum arabic and Aloe vera gel based edible coatings in combination with plant extracts on postharvest quality and storability of gola guava fruits, https://doi.org/10.1016/j.scienta.2020.109506, Sci. Hortic, 271(sept), 109506, (2020)

Azzolini, M., Jacomino, A. y otros tres autores, Ripening of Pedro Sato guava: study on its climateric or non-climateric nature, https://doi.org/10.1590/S1677-04202005000300004, Braz. J. Plant Physiol, 17(3), 299-306 (2005)

Arroyo, B., Bezerra, A. y otros cuatro autores, Antimicrobial active edible coating of alginate and chitosan add $\mathrm{ZnO}$ nanoparticles applied in guavas (Psidium guajava L.), https://doi.org/10.1016/j.foodchem.2019.125566, Food Chem, 309(mar), 125566 (2020)

Balaguera-López, H., Martínez Cárdenas, C. y Herrera Arévalo, A., Cambios durante la maduración del fruto de uchuva en condiciones agroecológicas de Venaquemada, Boyacá, Colombia, https://doi.org/https://doi.org/10.47796/ing.v2i01.298, Ing. Invest, 2(1), 286-293 (2019)

Bashir, A. y Abu-Goukh, A., Compositional changes during guava fruit ripening, https://doi/10.1016/S0308-8146(02)00345X, Food Chem, 80(4), 557-563 (2003)

Becerra, M., Pereira, A. y otros tres autores, Evaluación de la calidad de la guayaba, variedad enana roja EEA-1-23, durante el almacenamiento a temperatura ambiente, Rev. Cienc. Téc. Agropecu, 18(2), 70-73 (2009)

Biswas Murmu, S. y Niwas Mishra, H., Post-harvest shelf-life of banana and guava: mechanisms of common degradation problems and emerging counteracting strategies, https://doi.org/10.1016/j.ifset.2018.07.011, Innov. Food Sci. Emerg. Technol, 49(oct), 20-30 (2018)

Castellano, G., Quijada, O., Ramírez, R. y Sayago, E., Comportamiento poscosecha de frutas de guayaba (Psidium guajava L.) tratados con cloruro de calcio y agua caliente a dos temperaturas de almacenamiento, Rev. Iber. Tecnología Postcosecha, 6(2), 78-82 (2005)

Cavalini, F., Jacomino, A. y otros tres autores, Maturity indexes for "Fumagai" and "Paluma" guava. Rev. Bras. Frutic, ISSN: 1806-9967, 176-179 (2006)

de Oliveira, S., Mesquita, C. y otros seis autores, Improvement in physicochemical characteristics, bioactive compounds and antioxidant activity of acerola (Malpighia emarginata D.C.) and guava (Psidium guajava L.) fruit by-products fermented with potentially probiotic lactobacilli, https://doi.org/10.1016/j.Iwt.2020.110200, LWT, 134(dec), 110200 (2020)

Francisco, B., Pellá, M. y otros seis autores, Shelf-life of guavas coated with biodegradable starch and cellulose-based films, https://doi.org/10.1016/j.ijbiomac.2020.02.249, Int. J. Biol. Macromol, 152, 272-279 (2020)

Figueroa, J., Salcedo, J. y otros tres autores, Recubrimientos comestibles en la conservación del mango y aguacate, y perspectiva, al uso del propóleo en su formulacion, Rev. Colombiana Cienc. Anim. - Recia, 386-400 (2011)

Formiga, A., Pinsetta, J. y otros tres autores, Use of edible coatings based on hydroxypropyl methylcellulose and beeswax in the conservation of red guava Pedro Sato, https://doi.org/10.1016/j.foodchem.2019.03.142, Food Chem, 290(dec), 144$151(2019)$

García-Mera, G., Salas-Macías, C., y Canales-Torres, H., Recubrimiento comestible natural con base en Aloe vera como estrategia de conservación de Psidium guajava, https://doi.org/10.14483/23448350.11790, Rev. Cien, 3(30), 224 (2017)

González, I., Caracterización química del color de diferentes variedades de guayaba ( $P$. guajava L.) colombiana, Tesis de maestría, Departamento de Química, Facultad de Ciencias, Universidad Nacional de Colombia, Bogotá (2010)

González, R., Cervantes, Y. y Caraballo, L., Conservación de la guayaba (Psidium guajava L.) en postcosecha mediante un recubrimiento comestible binario, https://doi.org/10.21897/rta.v21i1.891, Temas Agrarios, 21(1), 54-64 (2016)

Gol, N., Patel, R. y Ramana, T., Improvement of quality and shelf-life of strawberries with edible coatings enriched with chitosan, https://doi.org/10.1016/j.postharvbio.2013.06.008, Postharvest Biol. Technol, 85, 185-195 (2013) 
Gutiérrez Guzmán, N., Dussan Sarria, S., y Castro Camacho, J., Fisiología y atributos de calidad de la guayaba "pera" (Psidium guajava cv.) en postcosecha, Revista de Ingeniería, 37, 26-30 (2012)

Nascimento, J., Stamford, T. y otros siete autores, Chitosan-citric acid edible coating to control Colletotrichum gloeosporioides and maintain quality parameters of fresh-cut guava, https://doi.org/10.1016/j.ijbiomac.2020.07.067, Int. J. Biol. Macromol, 163(nov), 1127-1135 (2020)

Hong, K., Xie, J. y otros tres autores, Effects of chitosan coating on postharvest life and quality of guava ( $P$ sidium guajava L.) fruit during cold storage, https://doi.org/10.1016/j.scienta.2012.07.002, Sci. Hortic, 144, 172-178 (2012)

Rehman, M., Asi, M., Hameed, A. y Bourquin, L., Effect of postharvest application of Aloe vera gel on shelf life, activities of anti-oxidative enzymes, and quality of gola guava fruit, https://doi.org/10.3390/foods9101361, Foods, 9(10), 1361 (2020)

Restrepo-Sánchez, D., Narváez-Cuenca, C. y Restrepo-Sánchez, L., Extracción de compuestos con actividad antioxidante de frutos de guayaba cultivada en Vélez-Santander, Colombia, https://doi/10.1590/S0100-40422009000600030, Quim. Nova, 32(6), 1517-1522 (2009)

Silva, A., Cortés, C. y Yahuaca, B., Evolución de indicadores de calidad de guayaba almacenada en frigorífico. Rev. Des, 1(oct), 5-11 (2006)

Singh, S. y Pal, R., Controlled atmosphere storage of guava (Psidium guajava L.) fruit, https://doi.org/10.1016/j.postharvbio.2007.08.009, Postharvest Biol. Technol, 47(3), 296-306 (2008)

Solarte, M., Romero, H. y Melgarejo, L., Caracterización ecofisiológica de la guayaba de la hoya del río Suárez, Desarrollo de productos funcionales promisorios a partir de la guayaba, ( $P$. guajava L.) para el fortalecimiento de la cadena productiva, editorial Universidad Nacional de Colombia, 1, 25-56, Bogotá, Colombia, (2010)

Vignoni, L., Césari, R., Forte, M. y Mirabile, M., Determinación de indice de color en ajo picado, http://dx.doi.org/10.4067/S0718-07642006000600011, Inf. Tecnol, 17(6), 63-67 (2006) 